\title{
EWGLA XVII: the XVII Meeting of the Euro Working Group on Locational Analysis
}

\author{
Mercedes Landete
}

Received: 21 May 2010 / Accepted: 7 June 2010 / Published online: 17 June 2010

(C) Sociedad de Estadística e Investigación Operativa 2010

The XVII Meeting of the Euro Working Group on Locational Analysis (EWGLA XVI) was held in Elche, Spain, from September 17 to 19, 2008. The Euro Working Group on Locational analysis (EWGLA) is a Working Group devoted to Locational Analysis within EURO (webpage: http://ewgla.fc.ul.pt/). It was created during the first EURO Summer Institute, held at Brussels, July/August 1984, devoted to the subject of Location Theory. The main aim of starting up the Working Group was to provide a network of people working together in the field of Locational Analysis. Since its creation, the group holds yearly meetings organized by one of its members. Various countries have hosted the meeting: France, Yugoslavia, Greece, Belgium, Germany, United Kingdom, Portugal and Spain. Each time, the meeting is designed to provide a dynamic forum for critical interchange of ideas and experiences about locational analysis and related fields.

EWGLA XVII was a fruitful workshop: more than 80 participants coming from 12 different countries took part in the meeting and a total of 56 scientific papers were presented and discussed there. The fact that fifteen percent of the assistants were students evidence that locational analysis is an interesting field for people starting research and also that the EWGLA group is concerned about promoting interest in the location analysis field.

The topics presented at the meeting covered many different areas in locational analysis. Papers in this special issue represent the work discussed in EWGLA XVII. Robustness is the topic of the paper "Conditional Median as a Robust Solution Concept for Uncapacitated Location Problems" by W. Ogryczak. The papers "The Ordered Capacitated Facility Location Problem” by A.M. Rodríguez-Chía, J. Kalcsics,

M. Landete ( $\square)$

Universidad Miguel Hernández de Elche, 03202 Elche, Alicante, Spain

e-mail: landete@umh.es 
S. Nickel and J. Puerto, "Lagrangean Bounds for the Optimum Communication Spanning Tree Problem" by Ivan Contreras, E. Fernández and A. Marín and "Discrete location for bundled demand points" by A. Marín evolve discrete location problems. Geometrical issues are discussed in the paper "Weber Problems With High-Speed Lines" by M. Körner and A. Schöbel while transit systems are the subject of the paper "A Study of Travel Time Prediction Using Universal Kriging" by H. Miura. The papers "The Single Period Coverage Facility Location Problem: Lagrangean heuristic and column generation approaches" by Y. Hinojosa, M. Albareda-Sambola, E. Fernández and J. Puerto "Fuzzy Goal Programming Models for Analyzing Demand Coverage" by I. Giannikos and "A computational comparison of several formulations for the multi-period incremental service facility location problem" by C. Pizarro, M. Albareda-Sambola, A. Alonso-Ayuso, L. Escudero, E. Fernández and Y. Hinojosa deal with covering and multi-period models. M. Ortega-Mier, M.C. Fonseca, A. García-Sánchez and F. Saldanha-da-Gama present a logistic approach in the paper "A Stochastic Bi-objective Location Model for Strategic Reverse Logistics". Daniel Scholz analyzes a continuous location model in his paper "The Multicriteria Big Cube Small Cube Method". Applications of location problems can be found in the paper "An Agent-Based framework for modeling and solving Location Problems" by A. Sgalambro, G. Bruno and A. Genovese. Two heuristic algorithms are introduced in the papers "A model and two heuristic approaches for a forage harvester planning problem: a case study" by B.V. Casas-Méndez, L. Carpente, C. Jácome and J. Puerto and "An exact procedure and LP formulations for the leader-follower location problem" by J.A. Moreno, C.M. Campos and D.R. Santos. Summing up, theoretical as well as practical issues are addressed in this special issue devoted to EWGLA XVII.

Apart from the regular talks, two plenary lectures were delivered by Silvano Martello from the University of Bologna, Italy and Rommert Dekker from the Erasmus University Rotterdam, Netherlands. Silvano Martello presented the talk "Assignment problems: The first fifty years":

In 1955 H.W. Kuhn published the Hungarian algorithm, the first polynomial-time algorithm for the assignment problem. In the next fifty years assignment problems attracted hundreds of researchers. Their studies accompanied and sometimes anticipated the development of Combinatorial Optimization, producing fundamental contributions to all algorithmic techniques in use nowadays. This talk was based on the book Assignment Problems by R. Burkard, M. Dell'Amico and S. Martello. S. Martello reviews the most important assignment problems (linear, quadratic, bottleneck) and the most relevant results in this area.

Rommert Dekker analyzed "Location models in reverse and service logistics":

While service logistics concerns all logistic activities after the purchase of a product, reverse logistics deals with all activities after the products have discarded by their users. Both fields have received increasing attention in the last decades, because of a lot of reasons. In both areas networks have to be set up and location models can be of use in that respect. In this talk, R. Dekker first outlined the specific aspects of both fields. These include uncertainty, less control, responsiveness issues, multiple players and the relation with inventories. Next he gave an overview of the location models set up for them and their applications. He dealt with mathematics aspects as well the impacts on economical geography. Finally he also discussed the integration between reverse and forward networks. 
Specially prepared for this especial issue of TOP is the invited paper "Routing problems with loading constraints" by Manuel Iori and Silvano Martello. Vehicle routing problems are among the most applicable and applied models of operations research in the economically important area of logistics. Both Manuel Iori and Silvano Martello are experts on the field of study. Their survey offers a wealth of bibliographic information as well as comparative computational results. They tackle interesting and important problems arising in real-world distribution systems and concerning the routing of vehicles in the presence of two- and three-dimensional loading constraints. Then, we have the pleasure to count with top researchers in routing problems for discussing the mentioned invited paper. Alphabetically ordered, we can read the comments of Gilbert Laporte, Frank Plastria, Juan José Salazar, Paolo Toth and José F. Oliveira.

EWGLA XVII was the first EWGLA meeting under the auspices of the present EWGLA board. It marked the end of a long, fruitful and dedicated coordination of EWGLA by Frank Plastria. Frank was the EWGLA coordinator from 1994 to 2007. He supervised the first web page of the group, coordinated 10 EWGLA meetings and has always insisted on the interaction of their members. Near to his 60th birthday all the locators wanted to make profit of the meeting for thanking Frank his invaluable work. The assistants to the meeting honored Professor Frank Plastria on behalf of all the location community for his notorious work and dedication in pro of EWGLA. A small book with a collection of EWGLA photos was the chosen gift.

Finally, I would like to express my gratitude. I would like to thank the sponsors for their support: we have received support from the Spanish Government, from the University Miguel Hernandez, from the Instituto Universitario Centro de Investigación Operativa, from the Spanish Red de Localización and from the EURO society. I want to thank all my colleagues from the organizing committee (J. Alcaraz, F.J. Toledo, J.F. Monge, M. Pulido, M.A. Marhuenda) because of their priceless help. I'd also like to give thanks to the members of the Scientific committee (L.F. Escudero, E. Fernández, I. Giannikos, M. Labbé, A. Marín, S. Nickel, M. Ndiaye, F. Plastria, J. Puerto, F. Saldanha da Gama) for their disposition and specifically to the EWGLA board (A. Marín, S. Nickel, F. Saldanha da Gama) who have really been involved in the organization of this meeting. Moreover I would like to thank our two invited speakers Prof Silvano Martello and Prof Rommert Dekker for their majestic talks and the time we exchanged. I'm also grateful for the support TOP and its editors, Emilio Carrizosa and Justo Puerto, have given me. I thank all participants in EWGLA XVII and all the reviewers who have made true this especial issue careful reading the submitted papers. 